\title{
Ação carrapaticida sobre Rhipicephalus microplus dos extratos, frações e compostos obtidos da espécie Lecythis lurida (Lecythidaceae)
}

\author{
Sinval Garcia Pereira ${ }^{1 *}$ \\ José Ribamar Silva do Nascimento Júnior ${ }^{1}$ \\ Aldilene da Silva Lima ${ }^{1}$ \\ Livio Martins Costa Junior ${ }^{1}$ \\ Giselle Maria Skelding Pinheiro Guilhon ${ }^{2}$ \\ Lourivaldo da Silva Santos ${ }^{2}$ \\ ${ }^{1}$ Universidade Federal do Maranhão, Centro de Ciências Agrárias e Ambientais \\ Coordenação de Ciências Biológicas, Campus IV \\ BR 222, km 04, Bairro Boa Vista, CEP 65.000.000, Chapadinha - MA, Brasil \\ ${ }^{2}$ Universidade Federal do Pará, Programa de Pós-Graduação em Química \\ Instituto de Ciências Exatas e Naturais, Campus Universitário do Guamá - Campus Básico \\ Rua Augusto Corrêa, 1, CEP 66075-110, Belém do Pará - PA, Brasil. \\ * Autor para correspondência \\ sinval@ufma.br \\ sinval.garcia@bol.com.br
}

Submetido em 17/04/2015

Aceito para publicação em 14/09/2015

\section{Resumo}

O Rhipicephalus microplus destaca-se entre os ectoparasitas de maior importância econômica na bovinocultura. O controle do carrapato bovino tem sido dificultado pela resistência de populações aos acaricidas sintéticos. Dessa forma, o objetivo do trabalho foi avaliar a eficiência carrapaticida de extratos, frações e metabólitos secundários de Lecythis lurida no combate ao $R$. microplus. Esse é o primeiro relato de estudo químico e biológico da espécie L. lurida, conhecida no cerrado leste do Maranhão, onde foi coletada, como sapucarana. Destaca-se pela sua densidade populacional e também pela exuberância de sua floração, muito visitada por várias espécies de abelhas. A investigação química revelou que os compostos obtidos da espécie $L$. lurida se agrupam na classe dos triterpenos, entre eles o friedelanol e a friedelina, esse último, como composto majoritário, além de misturas de triterpenos e esteroides. A avaliação da atividade carrapaticida sobre larvas e fêmeas ingurgitadas de carrapatos da espécie $R$. microplus revelou que apenas alguns extratos e frações apresentaram atividade moderada, considerando como parâmetros as concentrações testadas dos extratos e frações obtidos a frio e a quente de folhas e cascas de L. lurida.

Palavras chaves: Carrapato; Fitoquímica; Plantas do Maranhão; Triterpeno

\section{Abstract}

Acaricide action on Rhipicephalus microplus of extracts, fractions and compounds obtained from Lecythis lurida (Lecythidaceae). Rhipicephalus microplus (cattle tick) stands out as one of the most economically 
important ectoparasites of cattle, but controlling this species has been difficult because populations are resistant to synthetic acaricides. The goal of this study was to evaluate the efficiency of Lecythis lurida extracts, fractions and secondary metabolites against $R$. microplus. This is the first report of a phytochemical and biological study of $L$. lurida, which was collected in the cerrado of eastern Maranhão where it is known as sapucarana. In this region, $L$. lurida stands out for its population density and flowers, which are commonly visited by several bee species. The compounds obtained from $L$. lurida during this study were triterpenoids, such as friedelanol and friedelin (the major compound), in addition to mixtures of triterpenes and steroids. The evaluation of acaricide activity on larvae and engorged ticks of $R$. microplus revealed that only some of the extracts and fractions, obtained from the leaves and bark of L. lurida, had moderate activity.

Key words: Phytochemistry; Plants of Maranhão; Tick; Triterpene

\section{Introdução}

O carrapato Rhipicephalus microplus constitui-se um dos maiores problemas parasitários enfrentados pela pecuária bovina, reduzindo ganho de peso, produção de bezerros, produção de leite e o valor do couro (ALONSO et al., 1992; GRISI et al., 2002; 2014). Além disso, o R. microplus é responsável pela transmissão de hemoparasitos causadores da "tristeza parasitária" (Babesia spp. e Anaplasma ssp.) (SOUSA et al., 2011). O controle do carrapato bovino é geralmente realizado utilizando acaricidas sintéticos, e o manejo inadequado, esquemas de tratamentos não planejados, uso de formulações caseiras e utilização indiscriminada (FURLONG et al., 2002; FAZA et al., 2013), vêm acelerando a seleção de carrapatos resistentes aos princípios ativos disponíveis comercialmente (GRAF et al., 2004; DOMINGUES et al., 2014).

O surgimento de populações de carrapatos $R$. microplus resistentes aos carrapaticidas comerciais é um problema observado em muitas propriedades rurais, gerando um prejuízo de US\$ 7 bilhões no mundo. Entretanto, os produtos carrapaticidas ainda são a melhor opção para o controle de carrapatos. A escolha e o uso correto dos princípios ativos são importantes fatores que determinam a ocorrência de populações de R. microplus resistentes (FIDELIS JUNIOR et al., 2014). Com o aumento da resistência a drogas antiparasitárias, a indústria tem hesitado em investir na pesquisa de novos produtos químicos, promovendo assim a busca por alternativas aos produtos sintéticos (CHAGAS et al., 2002).

Os produtos de origem vegetal podem ser uma alternativa de grande importância em substituição aos acaricidas sintéticos (SCHENKEL et al., 2003; BROGLIO-MICHELETTI et al., 2009). Atualmente, em diversos países os fitoterápicos representam uma alternativa aos altos custos dos fármacos sintéticos (TUROLLA; NASCIMENTO, 2006). As plantas além de desempenharem ações como antimicrobianas, antivirais, fungicidas, e inseticidas (CHAGAS et al., 2012), possuem vantagens como suprimento sustentável, baixo custo, e biodegradabilidade (HEIMERDINGER et al., 2006). Deste modo, acredita-se que o uso de extratos vegetais de uma forma isolada ou associada pode provocar um desenvolvimento bem mais lento da resistência (ROEL, 2001).

A família Lecythidaceae possui distribuição pantropical, com maior concentração na região neotropical (SOUZA et al., 2001; HUANG et. al., 2015) e formam possivelmente um táxon monofilético (MORI et al., 2007), incluindo cerca de 25 gêneros e 300 espécies. No Brasil são registrados apenas 14 gêneros, sendo o gênero Lecythis o mais comum e caracterizase pela peculiaridade de seus frutos na forma de urnas, de casca dura e de aparência lenhosa, e que produzem uma boa quantidade de amêndoas comestíveis ou não (SOUZA; LORENZI, 2005).

$\mathrm{Na}$ família Lecythidaceae, destacam-se os metabólitos secundários pertencentes à classe dos terpenos como, friedelina, friedelanol, $\alpha$-amirina, $\beta$-amirina, cinamato de $\alpha$-amirina, cinamato de $\beta$-amirina, $\alpha$-amirenona, $\beta$-amirenona, lupeol, taraxasterol, juntamente com sitosterol e estigmasterol e também $\alpha$-tocoferol e tocotrienol saponinas triterpênicas (CARVALHO et al., 1998; CRUBLET et al., 2002; ALMEIDA et al., 2011). 
Em estudos biológicos de espécies da família Lecythidaceae foram demonstradas ações antiartrítica, antifúngica, antiprurítica (FRANCO; BARROS, 2006; HUSSIN et al., 2009; PATIL et al., 2011), cicatrizante de feridas tópicas (McRAE et al., 2008) e as folhas, flores e cascas da espécie Couroupita guianensis (espécie popularmente conhecida no Brasil como abricó-demacaco) são usadas na América do Sul na forma de chás ou infusões para tratar dor e inflamação (PINHEIRO et al., 2010).

A espécie Lecythis lurida ocorre ao longo da costa brasileira do Rio de Janeiro a Pernambuco e na Amazônia Oriental, em florestas maduras não sujeitas a alagamento, florestas secundárias e cerrados, apresentando menor estatura nesses dois últimos ambientes (MORI; PRANCE, 1981).

O presente estudo busca o embasamento científico inicial para o desenvolvimento de um fitoterápico para o combate ao carrapato dos bovinos. A planta L. lurida (sapucarana) é muito comum no município de Chapadinha - MA, localizado no cerrado leste do Maranhão e há relatos de sua utilização por pequenos criadores no combate aos ectoparasitos de caprinos. Dentre os objetivos destacam-se, avaliação da eficiência carrapaticida de extratos, frações e dos metabólitos secundários obtidos da $L$. lurida sobre larvas e fêmeas de carrapato da espécie $R$. microplus.

\section{Material e Método}

\section{Procedimentos gerais}

Os espectros de RMN 1D $\left({ }^{1} \mathrm{H},{ }^{13} \mathrm{C}\right.$ e $\left.1 \mathrm{D}-\mathrm{DEPT}\right)$ foram obtidos em espectrômetro VARIAN modelo MERCURY-300 - $300 \mathrm{MHz}$ ). Os deslocamentos químicos de $\mathrm{RMN}{ }^{1} \mathrm{H} \mathrm{e}{ }^{13} \mathrm{C}$ foram expressos em ppm $(\delta)$ em relação ao sinal do TMS, como referência interna, e as constantes de acoplamento $(J)$ em Hz.

No fracionamento por cromatografia em coluna (CC), utilizou-se sílica gel 0,063-0,2 mm (Vetec) como fase estacionária em coluna de vidro e sistema eluente, iniciando com hexano $100 \%$ seguido de mistura de hexano-acetato de etila com aumento da polaridade em escala de 5\%, até chegar ao acetato de etila $100 \%$ e, posteriormente a mistura acetato de etila/metanol, também com aumento de polaridade em escala de $5 \%$ e por último com metanol $100 \%$. Os constituintes majoritários foram obtidos puros ou em misturas e confirmados pelos dados de RMN experimental e, em comparações com os dados da literatura.

A cromatografia em camada delgada comparativa (CCD) foi empregada para análise e reunião das subfrações obtidas por cromatografia em coluna, preparadas por uma suspensão de $30 \mathrm{~g}$ de sílica gel (60G-MERCK) com indicador de fluorescência com 60 $\mathrm{mL}$ de água destilada. As revelações das cromatoplacas foram feitas por câmara de análise de fluorescência por luz ultravioleta, cabine tipo SPECTROLINE (modelo CM-10. Luz tipo SPECTROLINE modelo ENF-260C) através da exposição destas à radiação ultravioleta (UV) nos comprimentos de onda 254 e $366 \mathrm{~nm}$ e por borrifamento com solução ácida de sulfato cérico $\left(\mathrm{CeSO}_{4}\right)$ em ácido sulfúrico $\left(\mathrm{H}_{2} \mathrm{SO}_{4}\right)$ /água 1:1 ou solução de $\mathrm{H}_{2} \mathrm{SO}_{4} / \mathrm{MeOH} 1: 1$, seguido de aquecimento por cinco minutos em estufa á temperatura de $70{ }^{\circ} \mathrm{C}$. O critério de pureza adotado para as substâncias isoladas foi à observação de uma única mancha em CCD.

\section{Material vegetal}

As folhas e cascas foram coletadas no Cerrado leste do Maranhão, localizado no município de Chapadinha - MA e, posteriormente, foram secas à temperatura ambiente, em local coberto e arejado e trituradas em moinho tipo faca. Uma exsicata da planta foi depositada no Herbário do Museu Paraense Emílio Goeldi em Belém - PA (MPEG): Lecythis lurida (Miers) S. A. Mori (MG 200419).

\section{Obtenção dos extratos, frações e constituintes químicos}

Na primeira etapa obtiveram-se os extratos apolares e polares, a frio e a quente e as frações orgânicas para estudo biológico. Utilizou-se $400 \mathrm{~g}$ do material (folhas e cascas) seco e triturado, com extrações a frio por maceração com hexano por sete dias, seguido de filtração em papel de filtro, concentração em evaporador rotativo, 
obtendo os extratos apolares: extrato hexânico de folhas (LFFA - 0,18 g) e extrato hexânico de casca (LCFA 0,16 g). Com o resíduo da etapa anterior, fez-se extração com metanol em maceração por sete dias, filtrou-se e concentrou-se em evaporador rotativo, obtendo-se os extratos polares: extrato metanólico de folhas (LFFP $0,16 \mathrm{~g}$ ) e extrato metanólico de casca (LCFP - 0,17 g). Os extratos metanólicos foram particionados utilizando uma parte de água destilada para três de acetato de etila
PA. Após concentração em evaporador rotativo foram obtidas as frações: fração obtida da partição do extrato metanólico de folhas (LFFI - 0,10 g) e fração obtida da partição do extrato metanólico de casca (LCFI - 0,8 g) (Figura 1).

A extração a quente foi realizada em banhomaria, em um balão de fundo redondo, adaptou-se um condensador de refluxo e extraiu-se primeiramente com hexano durante $2 \mathrm{~h}$ com temperatura máxima

FIGURA 1: Fluxograma de obtenção dos extratos apolares e polares a frio e a quente e das frações das folhas e cascas de Lecythis lurida.

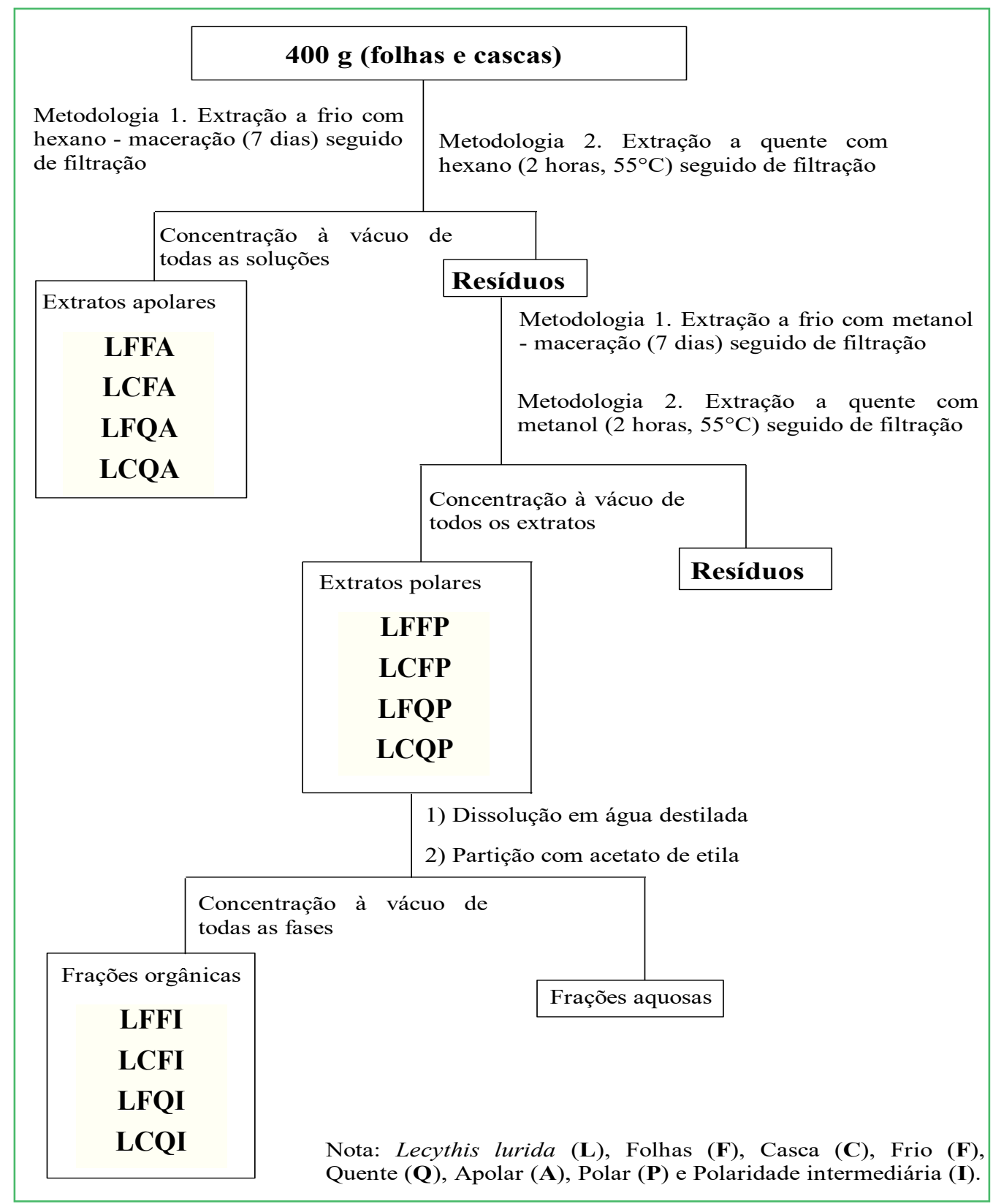


de $55{ }^{\circ} \mathrm{C}$. Filtrou-se e concentrou-se em evaporador rotativo obtendo os extratos apolares: extrato hexânico de folhas (LFQA - 0,19 g) e extrato hexânico de casca (LCQA - 0,08 g). Após evaporação do hexano extraiuse com metanol nas mesmas condições, obtendo-se os extratos polares: extrato metanólico de folhas (LFQP - 0,17 g) e extrato metanólico de casca (LCQP - 0,13 g). Os extratos metanólicos foram particionados utilizando uma parte de água destilada para três de acetato de etila PA. Após a concentração em evaporador rotativo obteve-se as frações: fração obtida da partição do extrato metanólico de folhas (LFQI - 0,8 g) e fração obtida da partição do extrato metanólico de casca (LCQI - 0,6 g). Em todas as extrações a massa do material botânico foi de $400 \mathrm{~g}$, totalizando doze extratos e frações, considerando as extrações a frio e a quente para estudo de atividade carrapaticida (Figura 1).

Para estudo químico utilizou-se $2.610 \mathrm{~g}$ de cascas de caule de L. lurida com extração a quente, em banhomaria, como descrito acima, obtendo-se o extrato apolar (LCQA - $121 \mathrm{~g}$ ), seguido pela obtenção do extrato polar (LCQP-68 g) e por último a fração orgânica (LCQI - $48 \mathrm{~g}$ ). As frações aquosas de todos os procedimentos citados acima foram descartadas. A fração LCQI $(19,6 \mathrm{~g})$ foi submetida à cromatografia em coluna (CC) e obteve-se 39 subfrações. Após o fracionamento, as subfrações obtidas foram monitoradas por CCD, sendo reunidas aquelas com perfis cromatográficos semelhantes e quando apresentava um composto majoritário era purificado com solventes adequados em função da polaridade ou realizava-se recristalização e filtração.

\section{Bioensaios de atividade carrapaticida}

\section{Infestação artificial de Rhipicephalus microplus em bezerros}

Bezerros mestiços, Holandês X Zebu, foram utilizados para obtenção da população de $R$. microplus. Cada bezerro foi infestado quinzenalmente com 4.000 larvas de carrapato e as fêmeas ingurgitadas foram coletadas após 21 dias da infestação. Durante as infestações os bezerros foram mantidos em baias com piso de cimento, com água e sal mineral à vontade, e alimentados com capim-elefante picado no cocho e ração balanceada. Este procedimento está registrado na Comissão de Ética de Uso Animal - CEUA/UFMA, sob protocolo, no 23115018061 / $2011-01$.

Para os testes de sensibilidade larvicida, as fêmeas ingurgitadas foram alocadas em estufas BOD, com temperatura de $27^{\circ} \mathrm{C}$ e umidade relativa $\geq 80 \%$, por 15 dias (período de coleta da postura), pesada em grupos de $0,2 \mathrm{~g}$ (aproximadamente 4.000 ovos) e acondicionada em seringas descartáveis modificadas para este uso. As seringas foram novamente levadas para a estufa, $27^{\circ} \mathrm{Ce}$ UR $\geq 80 \%$, por mais 15 dias, para a eclosão das larvas. Foram utilizadas, para o teste de sensibilidade larvar, larvas com idade entre 14 a 21 dias de eclosão.

\section{Sensibilidade larvar em papel impregnado}

O teste de sensibilidade foi realizado de acordo com a técnica desenvolvida por Stone e Haydock (1962) e adaptações da FAO (1971) e Leite (1988). Aproximadamente 100 larvas do carrapato foram colocadas entre dois papeis de filtro medindo $2 \times 2 \mathrm{~cm}$ $\left(4 \mathrm{~cm}^{2}\right)$, impregnados com $0,3 \mathrm{~mL}$ de cada concentração dos diferentes extratos, frações e compostos puros, os quais formavam um "sanduíche". Esse "sanduíche" foi colocado dentro de um envelope de papel filtro não impregnado de $8,5 \times 8,5 \mathrm{~cm}\left(72,25 \mathrm{~cm}^{2}\right)$ e vedado com pregadores plásticos, segundo FAO (1971). $\mathrm{O}$ envelope foi acondicionado em estufa a $27{ }^{\circ} \mathrm{C}$ e UR $\geq 80 \%$, durante $24 \mathrm{~h}$. Após este período, as larvas vivas e mortas foram contadas com a ajuda de um compressor a vácuo adaptado com uma pipeta, não sendo necessária a contagem quando todas as larvas estavam mortas $(100,0 \%$ de mortalidade) ou vivas $(0,0 \%$ de mortalidade). Foram utilizados quatro repetições para cada tratamento e nos controles foram utilizados os mesmos solventes (água destilada, soluções de álcool $70 \%$; triton 2\%; DMSO 3\% e tween 80 3\%) utilizados para preparação das soluções dos extratos e frações. Essas soluções foram testadas nas concentrações de 1,$0 ; 5,0 ; 10,0 ; 15,0$ e $20,0 \%$ os compostos puros foram testados em única concentração $(5,0 \%)$. Os resultados do teste de sensibilidade larvar foram corrigidos de acordo com a fórmula de Abbott (1925). 


\section{Sensibilidade das fêmeas ingurgitadas} em testes de imersão

O teste de sensibilidade de fêmeas ingurgitadas de $R$. microplus foi realizado utilizando o teste de imersão de acordo com a técnica desenvolvida por Drummond et al. (1973). As fêmeas ingurgitadas de R. microplus coletadas dos bezerros infestados artificialmente foram lavadas em água corrente, secas em papel toalha e pesadas em grupos de 10 espécimes, com grupos mais homogêneos possíveis. Foram testadas as concentrações de 1,$0 ; 5,0 ; 10,0 ; 15,0 ;$ e 20,0\% de extratos e frações.

Cada grupo de carrapato foi submerso, durante cinco minutos, nas diferentes concentrações dos extratos e frações da planta. Após este período, as fêmeas ingurgitadas foram secas em papel toalha, acondicionadas em placas de Petri e levadas a estufa BOD a $27^{\circ} \mathrm{C}$ e UR $\geq 80 \%$, por 15 dias, quando foram coletadas, pesadas as posturas e acondicionadas em seringas descartáveis modificadas para este uso. As seringas foram acondicionadas novamente para a estufa, $27^{\circ} \mathrm{C}$ e UR $\geq 80 \%$, por mais 15 dias, para a eclosão das larvas, quando foi avaliado visualmente o percentual de eclosão. Nos controles foram utilizados os mesmos solventes (água destilada, soluções de álcool 70\%; triton 2\%; DMSO 3\% e tween 80 3\%) utilizado para preparação das soluções dos extratos e frações. Os dados como Peso (massa) das Teleóginas (PT), Peso (massa) dos Ovos (PO) e Percentual de Eclosão (\%E), foram avaliados segundo as fórmulas prescritas por Drummond et al. (1973), para o cálculo da Eficiência Reprodutiva (ER) e da Eficiência do Produto (EP) em percentual.

\section{Análise estatística}

O delineamento experimental foi inteiramente casualizado, com quatro repetições. Aos dados aplicou à técnica estatística Análise de Variância (ANOVA) para verificação estatística dos efeitos carrapaticidas, como os dados satisfizeram as pressuposições, utilizou-se regressão linear simples. Foram utilizados os softwares
SPSS 15.0 e BIOESTAT 4.0 e o Excel para a formação e formalização das tabelas.

\section{Resultados}

\section{Estudo fitoquímico}

No estudo químico, foram obtidos os compostos puros, friedelanol (1) e a friedelina (2) e mistura dos triterpenos, taraxerol (3), $\alpha$-amirina (4), $\beta$-amirina (5) e lupeol (6) e também a mistura de esteroides, sitosterol (7) e estigmasterol (8) (Figura 2).

\section{Ação carrapaticida dos extratos, frações e dos constituintes químicos}

Todos os extratos e frações obtidos foram testados sobre larvas de $R$. microplus, nas concentrações de 1,0; 5,$0 ; 10,0 ; 15,0$ e $20,0 \%$, contudo, são apresentados na Tabela 1 e na Figura 3 os dados mais expressivos, entre eles, o extrato obtido a frio das folhas (LFFP), com concentrações letais $\left(\mathrm{CL}_{50}\right)$ de $4,21 \%$ e $7,08 \%$ para larvas e fêmeas, respectivamente. Nesse estudo, apenas a frações LCFI apresentou uma eficiência moderada, sendo que os demais extratos e frações apresentaram atividade carrapaticida abaixo de $12,0 \%$. A atividade carrapaticida sobre fêmeas ingurgitadas de $R$. microplus foi verificada, utilizando-se os extratos e frações com os melhores percentuais larvicidas, sobre as larvas $R$. microplus. Os extratos testados foram LCQP, LFFP, LFQP e a fração LFQI que apresentaram resultados próximos aos observados para as larvas da mesma espécie. Observou-se também que a utilização de diferentes temperaturas na obtenção dos extratos não influenciou a atividade carrapaticida.

Nesse trabalho também foram testados os metabólitos secundários obtidos puros ou em misturas (Figura 2) em concentração única de $5,0 \%$ os quais não apresentaram ação carrapaticida nas concentrações testadas sobre larvas de $R$. microplus. 
FIGURA 2: Estruturas dos metabólitos secundários obtidos da espécie Lecythis lurida.

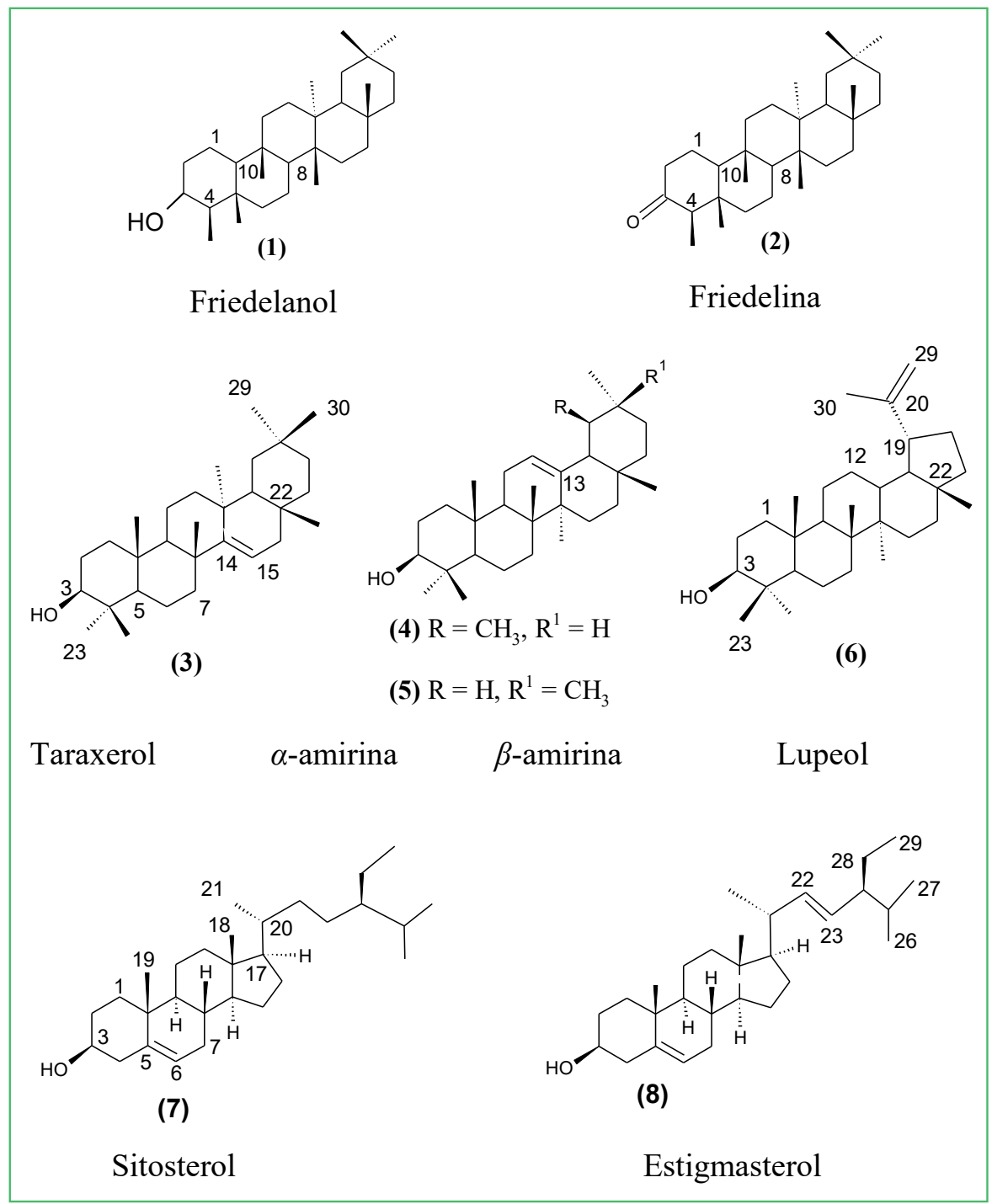

TABELA 1: Dados de regressão linear com os parâmetros de eficiência carrapaticida dos tratamentos com os extratos e frações mais eficientes sobre larvas e fêmeas de Rhipicephalus microplus, em relação ao tratamento testemunha.

\begin{tabular}{cccccc}
\hline & \multicolumn{5}{c}{ Ação carrapaticida (\%) } \\
\hline Extratos e frações (\%) & & $\mathbf{R}^{\mathbf{2}}$ & $\mathbf{C L}_{\mathbf{5 0}}$ & $\mathbf{L C}_{\mathbf{9 5 \%}}$ & Equação \\
\hline LFFP & Larva & 0,66 & 4,21 & 3,95 a 4,46 & $\mathrm{y}=4,51 \mathrm{x}+28,37$ \\
LFFP & Fêmea & 0,87 & 7,08 & 6,32 a 7,84 & $\mathrm{y}=3,89 \mathrm{x}+19,69$ \\
LFQP & Larva & 0,98 & 10,81 & 10,37 a 11,26 & $\mathrm{y}=5,50 \mathrm{x}-9,54$ \\
LFQP & Fêmea & 0,87 & 21,82 & 21,37 a 22,27 & $\mathrm{y}=2,06 \mathrm{x}-8,29$ \\
LFQI & Larva & 0,79 & n.c. & n.c. & $\mathrm{y}=0,74 \mathrm{x}+2,69$ \\
LFQI & Fêmea & 0,78 & n.c. & n.c. & $\mathrm{y}=0,32 \mathrm{x}+9,19$ \\
LCQP & Fêmea & 0,97 & n. c. & n. c. & $\mathrm{y}=1,24 \mathrm{x}+0,52$ \\
LCFI & Larva & 0,99 & 12,19 & 11,56 a 12,87 & $\mathrm{y}=4,24 \mathrm{x}-1,43$ \\
\hline
\end{tabular}

LFFP: Extrato metanólico de folhas obtido a frio; LFQP: extrato metanólico de folhas obtido a quente; LFQI: fração obtida da partição do extrato metanólico de folhas obtido a quente; LCQP: extrato metanólico de casca obtido a quente; LCFI: fração obtida da partição do extrato metanólico de casca obtido a frio; $\mathrm{R}^{2}$ : regressão linear; $\mathrm{CL}_{50}$ : Concentração (\%) tóxica para 50\% das larvas de Rhipicephalus microplus; $\mathrm{LC}_{95 \%}$ : limite de confiança a $95 \%$ de probabilidade do $\mathrm{CI}_{50}$. n. c., não calculado. 
FIGURA 3: Gráficos de regressão linear com os parâmetros de atividade carrapaticida dos tratamentos com os extratos e frações mais eficientes sobre larvas e fêmeas de Rhipicephalus microplus, em relação ao tratamento testemunha.

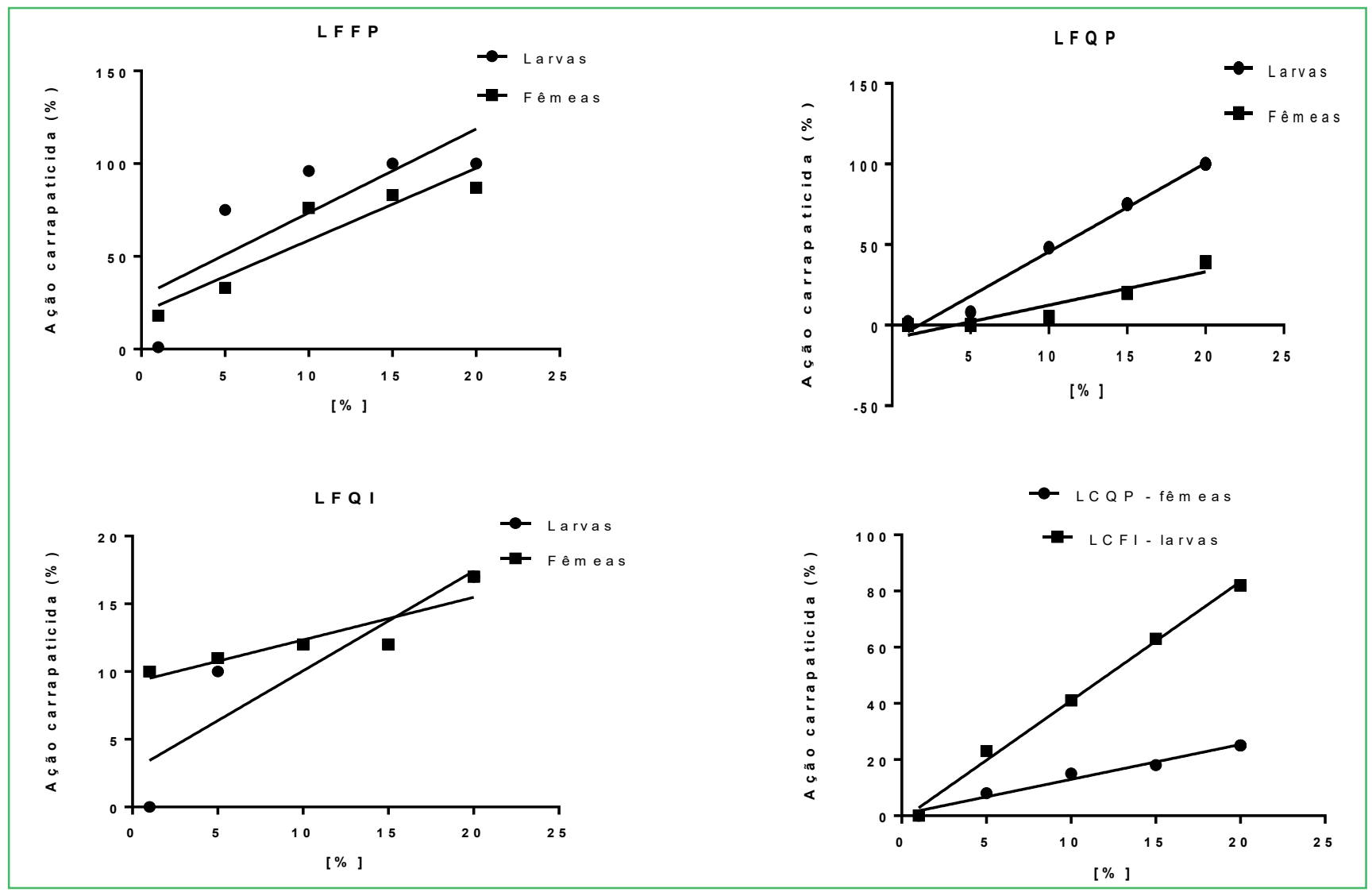

LFFP: Extrato metanólico de folhas obtido a frio; LFQP: extrato metanólico de folhas obtido a quente; LFQI: fração obtida da partição do extrato metanólico de folhas obtido a quente; LCQP: extrato metanólico de casca obtido a quente; LCFI: fração obtida da partição do extrato metanólico de casca obtido a frio.

\section{Discussão}

Esse é o primeiro relato de estudo químico e biológico da espécie L. lúrida. No estudo químico, os compostos obtidos se agrupam na classe dos triterpenos pentacíclicos, friedelanol e a friedelina, esse último como composto majoritário, além da mistura de triterpenos e esteroides. O estudo químico foi realizado com a fração LCQI (19,6 g) submetida a CC, da qual se obteve 39 subfrações e nestas foram identificadas duas substâncias puras, ambas na forma de cristais. As subfrações de 1 a 6 foram reunidas e purificadas e a massa final foi de $41 \mathrm{mg}$ $\mathrm{e}$, em seu espectro de $\mathrm{RMN}{ }^{1} \mathrm{H}$ observou-se vários sinais entre $\delta_{\mathrm{H}} 0,8$ e 1,7 característicos de sinais de hidrogênio metílicos de triterpeno, além de um simpleto largo em $\delta_{\mathrm{H}}$ 3,74 atribuído a um átomo de hidrogênio oximetínico. $\mathrm{O}$ conjunto de informações do espectro de $\mathrm{RMN}{ }^{1} \mathrm{H}$ mais os dados de $\mathrm{RMN}{ }^{13} \mathrm{C}$ (Tabela 2) possibilitaram sugerir a estrutura do friedelanol (1) (Figura 2), cujos dados estão de acordo com a literatura (ALMEIDA et al., 2011). Na reunião das subfrações de 7 a 15 obtevese a substância majoritária, com massa de $845 \mathrm{mg}$. O espectro de RMN ${ }^{1} \mathrm{H}$ dessa substância apresentou um conjunto de sete simpletos em $\delta_{H} 0,72 ; 0,86 ; 0,88 ; 0,95$; 0,$99 ; 1,04$ e 1,17 , indicando a presença de sete grupos metílicos na molécula, sugerindo tratar-se também de triterpeno pentacíclico. O espectro apresentou ainda um multipleto com sinais entre $\delta_{\mathrm{H}} 2,20$ e 2,39, indicando a presença de três hidrogênios alfa-carbonílicos, além de sinais entre $\delta_{\mathrm{H}} 1,25$ e 1,98, indicando a presença de hidrogênio metilênicos e metínicos na molécula. Os dados obtidos no espectro de $\mathrm{RMN}{ }^{13} \mathrm{C}$ (Tabela 2) mostraram sinais para de 30 átomos de carbono, com destaque para o sinal em $\delta_{\mathrm{C}} 213,68$, atribuído à carbonila 
em C-3. Os deslocamentos químicos estão de acordo com a estrutura da friedelina (2) (Figura 2) e foram comparados com os dados da literatura (ALMEIDA et al., 2011). Desse estudo obtiveram-se ainda substâncias em mistura, a primeira mistura foi obtida da reunião das subfrações de 16 a 24, uma mistura de triterpenos (40 mg), entre eles o taraxerol (3) (Figura 2), cujos sinais de $\mathrm{RMN}{ }^{1} \mathrm{H}$ destacam o $\delta_{\mathrm{H}} 5,53(d d, J=3,3$ e $8,1 \mathrm{~Hz}, 1 \mathrm{H})$ e em $\delta_{\mathrm{H}} 3,19(d d, J=5,1$ e $10,8 \mathrm{~Hz}, 1 \mathrm{H})$, característicos do hidrogênio H-15 e do H-3, respectivamente, e estão de acordo com a literatura (SOUZA et al., 2001). Ainda, como componente da mistura, a $\alpha$-amirina (4) e $\beta$-amirina (5) (Figura 2), cujos sinais no espectro de
$\mathrm{RMN}^{1} \mathrm{H}$ mais importantes são os dois tripletos atribuídos ao hidrogênio $\mathrm{H}-12$ em $\delta_{\mathrm{H}} 5,17(J=3,4 \mathrm{~Hz}, 1 \mathrm{H})$ e $\delta_{\mathrm{H}} 5,12(J=3,8 \mathrm{~Hz}, 1 \mathrm{H})$, respectivamente, também de acordo com Souza et al. (2001). E o outro componente da mistura é o lupeol (6) (Figura 2), no espectro de RMN ${ }^{1} \mathrm{H}$, observou-se os sinais em $\delta_{\mathrm{H}} 4,68(d, J=2,4 \mathrm{~Hz})$ e $\delta_{\mathrm{H}} 4,55(\mathrm{~m})$ referentes a hidrogênio da ligação dupla terminal (H-29) e a sobreposição dos sinais em $\delta_{\mathrm{H}} 3,20$, referente a H-3, estas informações estão de acordo com a literatura (CARVALHO et al., 1988; CURSINO et al., 2009). Somam-se as informações relatadas para os componentes da mistura os dados de $\mathrm{RMN}{ }^{13} \mathrm{C}$, apresentados na Tabela 2. Análise dessa mistura de

TABELA 2: Dados de $\mathrm{RMN}{ }^{13} \mathrm{C}\left(75 \mathrm{MHz}, \mathrm{CDCl}_{3}\right)$ das substâncias obtidas.

\begin{tabular}{|c|c|c|c|c|c|c|c|c|}
\hline \multirow[b]{2}{*}{$\mathrm{C}^{*}$} & \multicolumn{8}{|c|}{ RMN ${ }^{13} \mathrm{C}\left(\mathrm{CDCl}_{3}\right)$ das substâncias obtidas $\left(\mathrm{d}_{\mathrm{C}}\right)$} \\
\hline & (1) & (2) & (3) & (4) & (5) & $(6)$ & (7) & (8) \\
\hline 1 & 15,7 & 22,2 & 37,9 & 38,7 & 38,5 & 38,7 & 37,2 & 37,2 \\
\hline 2 & 36,0 & 41,5 & 27,0 & 27,2 & 26,8 & 27,4 & 29,7 & 29,7 \\
\hline 3 & 72,7 & 213,68 & 79,0 & 78,9 & 78,9 & 79,0 & 71,7 & 71,7 \\
\hline 4 & 49,1 & 58,19 & 39,2 & 38,7 & 38,7 & 38,7 & 39,7 & 39,6 \\
\hline 5 & 37,7 & 42,1 & 55,5 & 55,1 & 55,1 & 55,1 & 140,7 & 140,7 \\
\hline 6 & 41,6 & 41,2 & 18,7 & 18,3 & 18,3 & 18,3 & 121,6 & 121,6 \\
\hline 7 & 17,5 & 18,2 & 41,2 & 32,8 & 32,6 & 34,2 & 31,6 & 31,6 \\
\hline 8 & 53,1 & 53,06 & 38,7 & 39,9 & 39,7 & 40,8 & 31,8 & 31,8 \\
\hline 9 & 37,0 & 37,4 & 48,6 & 47,6 & 47,5 & 50,4 & 50,0 & 50,0 \\
\hline 10 & 61,3 & 59,42 & 37,6 & 36,8 & 36,9 & 37,1 & 36,1 & 36,4 \\
\hline 11 & 35,3 & 35,5 & 17,4 & 23,2 & 23,5 & 20,9 & 21,0 & 21,0 \\
\hline 12 & 30,6 & 30,4 & 35,4 & 124,3 & 121,6 & 25,1 & 39,7 & 39,6 \\
\hline 13 & 38,3 & 39,6 & 38,1 & 139,5 & 145,1 & 38,0 & 42,2 & 42,2 \\
\hline 14 & 39,6 & 38,2 & 158,0 & 41,5 & 41,6 & 42,8 & 56,7 & 56,6 \\
\hline 15 & 32,2 & 32,7 & 116,8 & 28,7 & 26,1 & 27,4 & 24,2 & 24,3 \\
\hline 16 & 35,5 & 35,9 & 36,6 & 26,5 & 27,1 & 35,5 & 29,0 & 28,2 \\
\hline 17 & 29,9 & 29,98 & 37,7 & 33,7 & 32,4 & 42,9 & 56,7 & 56,8 \\
\hline 18 & 42,7 & 42,7 & 49,2 & 59,0 & 47,1 & 48,2 & 11,9 & 12,1 \\
\hline 19 & 35,1 & 35,3 & 35,1 & 39,5 & 46,7 & 47,9 & 18,9 & 12,2 \\
\hline 20 & 28,1 & 28,1 & 28,7 & 39,6 & 31,0 & 150,9 & 36,1 & 40,4 \\
\hline 21 & 32,7 & 32,3 & 33,6 & 31,2 & 34,6 & 29,8 & 18,9 & 21,1 \\
\hline 22 & 39,2 & 39,2 & 33,0 & 41,5 & 37,1 & 39,9 & 33,7 & 138,2 \\
\hline 23 & 11,6 & 6,8 & 27,9 & 28,1 & 28,9 & 28,0 & 26,1 & 129,2 \\
\hline 24 & 16,3 & 14,6 & 15,7 & 15,6 & 15,5 & 15,4 & 45,7 & 51,2 \\
\hline 25 & 18,2 & 17,9 & 15,3 & 15,6 & 15,4 & 16,1 & 29,0 & 31,8 \\
\hline 26 & 20,1 & 20,2 & 29,8 & 16,8 & 16,7 & 15,9 & 19,3 & 21,1 \\
\hline 27 & 18,6 & 18,6 & 25,8 & 23,3 & 25,9 & 14,5 & 18,9 & 19,7 \\
\hline 28 & 32,0 & 32,0 & 29,7 & 28,7 & 28,3 & 18,0 & 23,0 & 25,9 \\
\hline 29 & 35,0 & 31,7 & 33,3 & 17,4 & 33,3 & 109,2 & 11,8 & 11,9 \\
\hline 30 & 31,7 & 35,0 & 21,2 & 21,3 & 23,6 & 19,3 & - & - \\
\hline
\end{tabular}

$\mathrm{C}^{*}$ : posições dos carbonos nas estruturas químicas. 
triterpenos por $\mathrm{RMN}{ }^{1} \mathrm{H} \mathrm{e}{ }^{13} \mathrm{C}$ mostrou a presença do taraxerol (3), a $\alpha$-amirina (4), $\beta$-amirina (5) e lupeol (6) na proporção de 14:46:30:10. A outra mistura obtida foi a de esteroides (37 mg), o sitosterol (7) e o estigmasterol (8) (Figura 2). No espectro de $\mathrm{RMN}{ }^{1} \mathrm{H}$ dessa mistura observou-se um multipleto em $\delta_{\mathrm{H}} 5,31$ atribuído a hidrogênio olefínicos do sitosterol e do estigmasterol no carbono C-5 das respectivas moléculas. No espectro de $\mathrm{RMN}{ }^{13} \mathrm{C}$ (Tabela 2) observou-se um sinal intenso em $\delta_{\mathrm{C}} 71,7$, característico de carbono carbinólico, atribuído ao C-3 em conformidade com a literatura (FACUNDO et al., 2008). Análise dessa mistura de esteroides por $\mathrm{RMN}{ }^{1} \mathrm{H} \mathrm{e}{ }^{13} \mathrm{C}$ mostrou a presença do sitosterol (7) e o estigmasterol (8) na proporção de 80:20.

Entre os extratos e frações testados, três extratos polares, o LFFP, o LFQP e o LCQP e duas frações, a LFQI E LCFI apresentaram atividade carrapaticida, todavia, essas atividades dependem de altas concentrações, tanto sobre as larvas como para as fêmeas ingurgitadas de carrapatos da espécie $R$. microplus. Muitas outras espécies de plantas investigadas demonstraram atividades carrapaticidas possibilitando efetivamente a utilização de extratos e metabólitos secundários em substituição ou mesmo em associação com os compostos sintéticos comerciais (CHAGAS et al., 2002; 2012). Zaman et al. (2012) observaram que os extratos aquosos de folhas de Azadirachta indica, Nicotiana tabacum, flores de Calotropis procera e sementes Trachyspermum ammi, apresentaram efeitos na diminuição da oviposição e no percentual de ecloção dos ovos e mortalidade de larvas de $R$. microplus. A ação do extrato aquoso de Eysenhardtia polystachya em carrapato $R$. microplus adultos e larvas demonstrou que o extrato não foi letal para as fêmeas ingurgitadas, contudo, teve um efeito significativo, inibindo em $64,58 \%$ a oviposição e foi altamente tóxico para as larvas, nos dois casos na concentração de $1 \%$. Esses resultados permitem sugerir o desenvolvimento de formulações larvicida com extratos da espécie E. polystachya (ALCALÁ et al., 2015). Sousa et al. (2011) avaliaram o efeito sinérgico de uma emulsão feita com frutos verdes de Melia azedarach com uma suspensão do fungo Beauveria bassiana no controle de R. microplus em bovinos artificialmente infestados. Essa associação proporcionou melhores resultados do que qualquer um dos dois tratamentos isolados, indicando a compatibilidade ou talvez uma sinergia entre $M$. azedarach e B. bassiana no controle do carrapato.

Estudos com capim-santo (Cymbopogon citrus) e $\operatorname{nim}($ A. indica) inibiram o tempo de pré-postura, tempo de postura e produção de ovos de fêmeas de carrapato das espécies R. microplus e Rhipicephalus sanguineus (SILVA et al., 2007).

Dados da literatura indicam que os compostos da classe dos triterpenos, em linhas gerais, não apresentam atividade larvicida. Montenegro et al. (2006) testaram e observaram que o taraxerol não apresentou atividade larvicida sobre Aedes aegypti nas concentrações analisadas. Em outra pesquisa ficou demonstraram que a friedelina, além de inativa, estimulou a proliferação dos parasitos de Leishmania guyanensis (ALMEIDA et al., 2011).

$\mathrm{Na}$ medicina tradicional, as folhas da espécie Lecythis pisonis são usadas como um banho para o tratamento de prurido no corpo humano (FRANCO; BARROS, 2006). E em estudos in vitro realizados por Silva et al. (2011) também demonstraram que extrato etanólico e frações (hexano, etérea, acetato de etila) de folhas de L. pisonis e também a mistura dos triterpenos, ácidos ursólico e oleanólico são capazes de suprimir o prurido no corpo de camundongos e de ratos administrado oralmente até a dose de $2 \mathrm{~g} \mathrm{~kg}^{-1}$.

Apesar dos extratos obtidos a frio e a quente de $L$. lurida não terem apresentado eficiência sobre larvas de carrapatos bovinos, outras plantas pertencentes à família Lecythidaceae apresentam componentes bioativos sobre fungos, apresentando inibição de até $70 \%$ do crescimento de fungos patogênicos, e ação antiinflamatória e antiartrítica (HUSSIN et al., 2009). Outros estudos feitos com plantas da mesma família utilizando extrato metanólico da casca obtiveram efeitos antiinflamatórios, antinociceptiva, antipirético (SANTOS et al., 2011) e bactericida (PRABHAKARAN et al., 2014).

Atualmente existe uma série de estudos realizados com produtos feitos a base de plantas e muitos deles têm identificado espécies vegetais com atividade biológica relevante. Estudos realizados com L. lurida são escassos na literatura e inexistentes sobre o controle do carrapato dos bovinos. Assim, é de fundamental 
importância o desenvolvimento de pesquisas que visem o conhecimento dessa espécie, principalmente no controle de patógenos.

\section{Agradecimentos}

Os autores agradecem a Universidade federal do Pará, ao Centro de Ciências Agrárias e Ambientais da Universidade Federal do Maranhão e a EMBRAPA pelo suporte de infraestrutura e a FAPESPA e FAPEMA pelo suporte financeiro.

\section{Referências}

ABBOTT, W. S. A method of computing the effectiveness of an insecticide. Journal of Economic Entomology, Riverside, v. 18, n. 1, p. 265-267, 1925.

ALCALÁ, Y.; RIVERO, F.; SUMANO, H.; GUTIÉRREZ, L. Acaricidal action of water extracts from Eysenhardtia polystachya against Rhipicephalus (Boophilus) microplus. Comparative Parasitology, Lawrence, v. 82, n. 1, p. 123-128, 2015.

ALMEIDA, M. de F. O; MELO, A. C. R. de; PINHEIRO, M. L. B.; SILVA, J. R. de A.; SOUZA, A. D. L. de S. Constituintes químicos e atividade leishmanicida de Gustavia elliptica (Lecythidaceae). Química Nova, São Paulo, v. 34, n. 7, p. 1182-1187, 2011.

ALONSO, M.; ARELLANO-SOTA, C.; CERESER, V. H.; CORDOVES, C. O.; GUGLIELMONE, A. A.; KESSLER, R.; MANGOLD, A. J.; NARI, A.; PATARROYO, J. H.; SOLARI, M. A. Epidemiology of bovine anaplasmosis and babesiosis in Latin America and the Caribbean. Revue Scientifique et Technique, Paris, v. 11, n. 3, p. 713-733, 1992.

BROGLIO-MICHELETTI, S. M. F.; VALENTE, E. C. N.; SOUZA, L. A. de; DIAS, N. da S.; ARAUJO, A. M. N. de. Extratos de plantas no controle de Rhipicephalus (Boophilus) microplus (Canestrini, 1887) (Acari: Ixodidae) em laboratório. Revista Brasileira de Parasitologia Veterinária, São Carlos, v. 18, n. 4, p. 44-48, 2009. CARVALHO, M. G. de; VELANDIA, J. R.; OLIVEIRA, L. F. de; BEZERRA, F. B. Triterpenos isolados de Eschweilera longipes Miers (Lecythidaceae). Química Nova, São Paulo, v. 21, n. 6, p. 740-743, 1998.

CHAGAS, A. C. de S.; BARROS, L. D. de; COTINGUIBA, F.; FURLAN, M.; GIGLIOTI, R.; OLIVEIRA, M. C. de S.; BIZZO, H. R. In vitro efficacy of plant extracts and synthesized substances on Rhipicephalus (Boophilus) microplus (Acari: Ixodidae). Parasitology Research, Heidelberg, v. 110, n. 1, p. 295-303, 2012.

CHAGAS, A. C. de S.; PASSOS, W. M.; PRATES, H. T.; LEITE, R. C.; FURLONG, J.; FORTES, I. C. P. Efeito acaricida de Eucalyptus em Boophilus microplus: óleos essenciais e concentrados emulsionáveis. Brazilian Journal of Veterinary Research and Animal Science, São Paulo, v. 39, n. 5, p. 247-253, 2002.

CRUBLET, M.-L; POUNY, I; DELAUDE, C; LAVAUD, C. Acylated triterpenoid sapanins from the Stem Bark of Foetidia Africana. Journal of Natural Products, Columbus, v. 65, n. 11, p. 1500-1567, 2002.
CURSINO, L. M. de C.; MESQUITA, A. S. S.; MESQUITA, D. W. de O.; FERNANDES, C. C.; PEREIRA JUNIOR, O. L.; AMARAL, I. L. do; NUNEZ, C. V. Triterpenos das folhas de Minquartia guianensis Aubl. (Olacaceae). Acta Amazonica, Manaus, v. 39, n. 1, p. 181-186, 2009.

DOMINGUES, R.; WOHLRES-VIANA, S.; REIS, D. R.; TEIXEIRA, H. C.; FERREIRA, A. P.; GUIMARÃES, S. E.; PRATA, M. C.; FURLONG, J.; VERMEQUE, R. S.; MACHADO, M. A. Expression of immune response genes in peripheral blood of cattle infested with Rhipicephalus microplus. Genetics and Molecular Research, Ribeirão Preto, v. 13, n. 2, p. 4013-4021, 2014.

DRUMMOND, R. O.; ERNEST, S. E.; TREVINO, J. L.; GLADNEY, W. J.; GRAHAM, O. H. Boophilus annulatus and Boophilus microplus: laboratory tests for insecticides. Journal of Economic Entomology, Riverside, v. 66, n. 1, p. 130-133, 1973.

FACUNDO, V.A.; POLLLI, A. R.; RODRIGUES, R. V.; MILITÃO, J. S. L. T.; STABELLI, R. G.; CARDOSO, C. T. Constituintes químicos fixos e voláteis dos talos e frutos de Piper tuberculatum Jacq. e das raízes de P. Hispidum H. B. K. Acta Amazonica, Manaus, v. 38, n. 4, p. 733-742, 2008.

FAO-PLANT PROTECTION BULLETIN. Recommended methods for the detection and measurement of resistance of agricultural pests to pesticides. Tentative methods for larvae of cattle tick Boophilus spp. FAO method, Cincinnati, v. 19, n. 7, p. 15-18, 1971.

FAZA, A. P.; PINTO, I. S.; FONSECA, I.; ANTUNES, G. R.; MONTEIRO, C. M.; DAEMON, E.; MUNIZ, M. de S.; MARTINS, M. F.; FURLONG, J.; PRATA, M. C. A new approach to characterization of the resistance of populations of Rhipicephalus microplus (Acari: Ixodidae) to organophosphate and pyrethroid in the state of Minas Gerais, Brazil. Experimental Parasitology, Amsterdam, v. 134, n. 4, p. 519-23, 2013.

FIDELIS JUNIOR, O. L.; SAMPAIO, P. H.; TEIXEIRA, P. P. M.; ANDRÉ, M. R.; CADIOLI, F. A. Resistência do Rhipicephalus (Boophilus) microplus aos carrapaticidas: revisão de literatura. Nucleus Animalium, Ituverava, v. 6, n. 1, p. 99-110, 2014.

FRANCO, E. A. P.; BARROS, R. F. M. Uso e diversidade de plantas medicinais no Quilombo Olho D'água dos Pires, Esperantina, Piauí. Revista Brasileira de Plantas Medicinais, Botucatu, v. 8, n. 3, p. 78-88, 2006.

FURLONG, J.; COSTA-JUNIOR, L. M.; CHAGAS, A. C. S.; REIS, E. S. $\mathrm{CL}_{50}$ e $\mathrm{CL}_{90}$ dos extratos alcoólico e aquoso de nim indiano (Azadirachta indica) em larvas de Boophilus microplus. In: CONGRESSO BRASILEIRO DE PARASITOLOGIA VETERINÁRIA, 12, 2002, Rio de Janeiro. Anais... Rio de Janeiro: Universidade Federal Rural do Rio de Janeiro, 2002. CD-Rom.

GRAF, J. F.; GOGOLEWSKI, R.; LEACH-BING, N.; SABATINI, G. A.; MOLENTO, M. B.; BORDIN, E. L.; ARANTES, G. J. Tick control: an industry point of view. Parasitology, London, v. 129, Suppl., p. 427- 442, 2004.

GRISI, L.; LEITE, R. C.; MARTINS, J. R. de S.; BARROS, A. T. M. de; ANDREOTTI, R.; CANÇADO, P. H. D.; LEÓN, A. A. P. de; PEREIRA, J. B.; VILLELA, H. S. Reassessment of the potential economic impact of cattle parasites in Brazil. Revista Brasileira de Parasitologia Veterinária, São Carlos, v. 23, n. 2, p. 150-156, 2014.

GRISI, L.; MASSARD, C. L.; MOYA B. G. E.; PEREIRA, J. B. Impacto econômico das principais ectoparasitoses em bovinos no 
Brasil. A Hora Veterinária, Porto Alegre, v. 125, n. 21, p. 8-10, 2002.

HEIMERDINGER, A.; OLIVO, C. J.; MOLENTO, M. B.; AGNOLIM, C. A.; ZIECH, M. F.; SCARAVELLI, L. F. B.; SKONISKI, F. R.; BOTH, J. F.; CHARÃO, P. S. Extrato alcoólico de Capim-cidreira (Cymbopogon citratus) no controle do Boophilus microplus em bovinos. Revista Brasileira de Parasitologia Veterinária, São Carlos, v. 15, n. 1, p. 37-39, 2006.

HUANG, Y.-Y.; MORI, S. A.; KELLY, L. M. Toward a phylogeneticbased generic classification of neotropical Lecythidaceae-I. Status of Bertholletia, Corythophora, Eschweilera and Lecythis. Bertholletia. Phytotaxa, Auckland, v. 203, n. 2, p. 85-121, 2015.

HUSSIN, N. M.; MUSE, R.; AHMAD, S.; RAMLI, J.; MAHMOOD, M.; SULAIMAN, M. R.; SHUKOR, M. Y. A.; RAHMAN, M. F. A.; AZIZ, K. N. K. Antifungal activity of extracts and phenolic compounds from Barringtonia racemosa L. (Lecythidaceae). African Journal of Biotechnology, Cape Town, v. 8, p. 28352842, 2009.

LEITE, R. C. Boophilus microplus (Canestrini, 1887): susceptibilidade, uso atual e retrospectivo de carrapaticidas em propriedades das regiões fisiogeográficas da baixada do GrandeRio e Rio de Janeiro. Uma abordagem epidemiológica. 1988. 151 f. Tese (Doutorado em Medicina Veterinária Preventiva) Universidade Federal de Minas Gerais, Belo Horizonte. 1988.

McRAE, J. M.; YANG, Q.; CRAWFORD, R. J.; PALOMBO, E. A. Antibacterial compounds from Planchonia careyaleaf extracts. Journal of Ethnopharmacology, Shannon, v. 116, n. 3, p. 554560,2008 .

MONTENEGRO, L. H. M.; OLIVEIRA, P. E. S.; CONSERVA, L. M.; ROCHA, E. M. M.; BRITO, A. C.; ARAÚJO, R. M.; TREVISAN, M. T. S.; LEMOS, R. P. L. Terpenóides e avaliação do potencial antimalárico, larvicida, anti-radicalar e anticolinesterásico de Pouteria venosa (Sapotaceae). Revista Brasileira de Farmacognosia, Curitiba, v. 16, Supl., p. 611-617, 2006.

MORI, S. A.; PRANCE, G. T. The "sapucaia" Group of Lecythis (Lecythidaceae). Brittonia, New York, v. 33, n. 1, p. 70-80, 1981.

MORI, S. A.; TSOU, C. H.; WU, C. C.; CRONHOLM, B.; ANDERBERG, A. A. Evolution of Lecythidaceae with an emphasis on the circumscription of neotropical genera: information from combined NDHF and TRNL-F sequence data. American Journal of Botany, St. Louis, v. 94, n. 3, p. 289-301, 2007.

PATIL, K. R.; PATIL, C. R.; JADHAV, R. B.; MAHAJAN, V. K.; PATIL, P. R.; GAIKWAD, P. S. Anti-arthritic activity of bartogenic acid isolated from fruits of Barringtonia racemosa Roxb. (Lecythidaceae). Evidence-Based Complementary and Alternative Medicine, New York, v. 5, n. 1, p. 112-117, 2011.

PINHEIRO, M. M. G.; BESSA, S. O.; FINGOLO, C. E.; KUSTER, R. M.; MATHEUS, M. E.; MENEZES, F. S.; FERNANDES, P. D. Antinociceptive activity of fractions from Couroupita guianensis Aubl. leaves. Journal of Ethnopharmacology, Shannon, v. 127, n. 2, p. 407-413, 2010.

PRABHAKARAN, M.; REEJO, B.; KUMAR, D. S. Antibacterial activity of fruit of Careya arborea Roxb. (Lecythidaceae). Hygeia: Journal for Drugs and Medicines, Tamil Nadu, v. 6, n. 12, p. 2024, 2014.

ROEL, A. R. Utilização de plantas com propriedades inseticidas: uma contribuição para o Desenvolvimento Rural Sustentável.
Revista Internacional de Desenvolvimento Local, Campo Grande, v. 1, n. 2, p. 43-50, 2001.

SANTOS, E. N.; LIMA, J. C. S. L.; NOLDIN, V. F.; CECHINELFILHO, V.; RAO, V. S. N.; LIMA, E. F. L.; SCHMEDAHIRSCHMANN, G.; SOUSA JR., P. T.; MARTINS, D. T. O. Anti inflammatory, antinociceptive, and antipyretic effects of methanol extract of Cariniana rubra stem bark in animal models. Anais da Academia Brasileira de Ciências, Rio de Janeiro, v. 83, n. 2, p. 557-566, 2011.

SCHENKEL, E. P.; GOSMANN, G; PETROVIC, P. R. Produtos de origem vegetal e o desenvolvimento de medicamentos. In: SIMÕES, C. M. O.; SCHENKEL, E. P.; GOSMANN, G.; MELLO, J. C. P.; MENTZ, L. A.; PETROVICK, P. R. (Org.). Farmacognosia: da planta ao medicamento. Cap. 15. 5. ed. Porto Alegre: UFRGS, Florianópolis: UFSC, 2003. p. 301-332.

SILVA, L. L.; GOMES, B. S.; SOUSA-NETO, B. P.; OLIVEIRA, J. P.; FERREIRA, E. L.; CHAVES, M. H.; OLIVEIRA, F. A. Effects of Lecythis pisonis Camb. (Lecythidaceae) in a mouse model of pruritus. Journal of Ethnopharmacology, Shannon, v. 139, n. 1, p. 90-97, 2011.

SILVA, W. W.; ATHAYDE, A. C. R.; RODRIGUES, O. G. ARAÚJO, G. M. B.; SANTOS, V. D.; NETO, A. B. S.; COELHO, M. C. O. C.; MARINHO, M. L. Efeitos do neem (Azadirachta indica A. Juss) e do capim santo [Cymbopogon citratus (DC) Stapf] sobre os parâmetros reprodutivos de fêmeas ingurgitadas de Boophilus microplus e Rhipicephalus sanguineus (Acari: Ixodidae) no semiárido paraibano. Revista Brasileira de Plantas Medicinais, Botucatu, v. 9, p. 1-5, 2007.

SOUSA, L. A. D.; PIRES JR., H. B.; SOARES, S. F.; FERRI, P. H.; RIBAS, P.; LIMA, E. M.; FURLONG, J.; BITTENCOURT, V. R. E. P.; PERINOTTO, W. M. de S.; BORGES, L. M. F. Potential synergistic effect of Melia azedarach fruit extract and Beauveria bassiana in the control of Rhipicephalus (Boophilus) microplus (Acari: Ixodidae) in cattle infestations. Veterinary Parasitology, Amsterdam, v. 175, n. 3-4, p. 320-324, 2011.

SOUZA, A. D. L de; ROCHA, A. F. I. Da; PINHEIRO, M. L. B.; ANDRADE, C. H. de S.; GALOTTA, A. L. de A. Q.; SANTOS, M. do P. S. S. Constituintes químicos de Gustavia augusta L. (lecythidaceae). Química Nova, São Paulo, v. 24, n. 4, p. 439-442, 2001.

SOUZA, V. C.; LORENZI, H. Botânica sistemática: guia ilustrativo para identificação das famílias de angiospermas da flora brasileira, baseado em APG II. Nova Odessa: Instituto Plantarum, 2005. 604 p.

STONE, B. F.; HAYDOCK, K. P. A method for measuring the acaricide susceptibility of the cattle Boophilus microplus (Can.). Bulletin of Entomological Research, Cambridge, v. 53, p. 563$578,1962$.

TUROLLA, M. S. dos R.; NASCIMENTO, E. de S. Informações toxicológicas de alguns fitoterápicos utilizados no Brasil. Revista Brasileira de Ciências Farmacêuticas, São Paulo, v. 42, n. 2, p. 289-306, 2006.

ZAMAN, M. A.; IQBAL, Z.; ABBAS, R. Z.; KHAN, M. N.; MUHAMMAD, G.; YOUNUS, M.; AHMED, S. In vitro and in vivo acaricidal activity of an herbal extract. Veterinary Parasitology, Amsterdam, v. 186, n. 3-4, p. 431-436, 2012. 